\title{
Study on Behavior of RPC Filled Steel Tubular Stub Columns under Axial Compression
}

\author{
S. H. Liu, ${ }^{\text {ab.c.c. }}$ L. H. Li, ${ }^{\text {b }}$ and L. Wang ${ }^{c}$ \\ a State Key Laboratory of Water Resources and Hydropower Engineering Science, Wuhan University, \\ Wuhan, China \\ ${ }^{b}$ School of Civil Engineering and Architecture, Hubei University of Technology, Wuhan, China \\ c Nanchang Key Laboratory of Material and Structure Detection, Jiangxi University of Technology. \\ Nanchang, China \\ ${ }^{1}$ shliu(@)whu.edu.cn
}

Reactive powder concrete (RPC) with compressive strength higher than $170 \mathrm{MPa}$ was prepared by using ordinary Portland cement, silica fume, ground granulated blast-furnace slag or fly ash. RPC shows high strength, constant Poisson's ratio, high compressive peak strain but post-peak brittle failure. Based on the RPC mechanical behavior study, investigation of the interaction between steel tubes and core RPC was performed, in view of its effect on the bearing capacity and deformability of RPC filled steel tubular (RPCFT) stub columns subjected to axial loading. RPCFT stub columns have very high ductility and alleviate the RPC disadvantage of brittle failure. However; the confinement effect of steel tubes on RPC is lower than that of normal strength concrete and thus, it can be neglected during the design process for convenience and safety.

Keywords: composite column, steel tubular, bearing capacity, load-strain behavior.

Introduction. Reactive powder concrete (RPC) is a new type ultrahigh performance concrete (UHPC) with a high compressive strength over $200 \mathrm{MPa}$ or more [1]. The use of RPC in construction can significantly increase the bearing capacity of structures and reduce element section sizes as well as structure deadweights. RPC becomes a highly dense cement-based composite with excellent strength and durability due to the elimination of aggregates larger than $1 \mathrm{~mm}$. RPC is applicable in long-span structures, high-rise buildings, structural repair, and unique elements for military purposes [2-4]. While RPC is a brittle material and post-peak brittle failure often occurs when it is subject to high compression level which will limit its application. Adding steel fibers can improve the tensile ductility of $\mathrm{RPC}$, but as indicated in this paper, steel fiber is not able to enhance the compressive ductility of RPC. Furthermore, the liquidity of fresh concrete is sharply reduced when the volumetric ratio of steel fiber exceeds $2 \%$.

Reinforced concrete column is a common compressive element. However, reinforced high strength concrete (HSC) columns have a fatal shortcoming that the cover layers often burst too early and cause premature bucking of reinforced bars even at the loads much lower than the HSC compressive strength $[5,6]$.

The ductility of RPC can be increased greatly when it is confined by a steel tube. The first application of RPC, the Sherbrooke pedestrian bridge took this structural formation [7]. However, although concrete filled steel tubular (CFT) column has been studied thoroughly, research regarding ultrahigh strength concrete filled steel tubular columns [8-11] is insufficient and far from immature.

During the past decades, steel tube-HSC combined columns have been successfully applied to many high-rise buildings. Though the RPC used in this study is a HSC without coarse aggregates, the conclusion derived by this study can be extended to the HSC with coarse aggregates as all types of HSC share some common properties irrespective of coarse aggregates. However, lacking related design standards is a problem at present. Most current 
standards mainly deal with concrete for the critical stress values below $60 \mathrm{MPa}$. To the best of the authors' knowledge, some organizations, such as Association Francaise de Genie Civil (AFGC), Japan Society of Civil Engineers (JSCE) and The International Federation for Structural Concrete (FIB) are now compiling HSC and UHPC related technical standards. The aim of this study is to offer a simple yet reliable engineering formula for reference before these standards are available.

1. Experimental. Raw materials used for RPC preparation are as follows: P. O. 42.5 ordinary portland cement, silica fume (SF), ground granulated blast-furnace slag (GS), fly ash (FA), polycarboxylate superplasticizer ( $40 \%$ solid content), tidy river sand (S) with particle diameters ranging from 0.2 to $0.4 \mathrm{~mm}$, and micro steel fiber (F) with a diameter of $0.2 \mathrm{~mm}$ and length of $13 \mathrm{~mm}$. Six types of RPC (C1-C6) were prepared. The mix proportions used are shown in Table 1.

$\mathrm{T}$ a b 1 e 1

RPC Mix Proportions $\left(\mathrm{kg} / \mathrm{m}^{3}\right)$ and Compressive Strength (MPa)

\begin{tabular}{|c|c|c|c|c|c|c|c|c|c|}
\hline Code & C & SF & GS & FA & W & S & F & $f_{\text {prism }}$ & $f_{\text {cub }}$ \\
\hline C1 & 745 & 132 & 219 & 0 & 175 & 1096 & 0 & 116 & 126 \\
C2 & 745 & 132 & 219 & 0 & 175 & 1096 & 37 & 118 & 134 \\
C3 & 884 & 221 & 0 & 0 & 155 & 1105 & 0 & 137 & 163 \\
C4 & 884 & 221 & 0 & 0 & 155 & 1105 & 150 & 145 & 178 \\
C5 & 737 & 184 & 0 & 184 & 155 & 1105 & 75 & 144 & 170 \\
C6 & 553 & 184 & 0 & 368 & 155 & 1105 & 150 & 147 & 172 \\
\hline
\end{tabular}

The RPC mixture was prepared using a counterflow pan mixer and was cast into molds. After demoulding, they were cured at $90^{\circ} \mathrm{C}$ in steam for three days. After heating treatment, the strength did not increase significantly anymore. Therefore, the strength was measured and regarded as the long-term strength (Table 1). Mechanical properties of RPC, such as compressive strength, 4-point bending strength parameters and Poisson's ratio are determined by the standard testing methods.

Steel tubes used in this study are seamless tubes with moderate to thick wall thickness. The preparation and curing of RPCFT specimens were similar to the methods of RPC specimens. Loading setup of RPCFT specimens is also illustrated in Fig. 1. Loading was controlled by displacement at the speed of $10 \mu \varepsilon / \mathrm{s}$ till the axial deformation rate of the columns reached about 5\%. Two displacement transducers with a $120 \mathrm{~mm}$ gauge length were used to record the axial compressive strain.

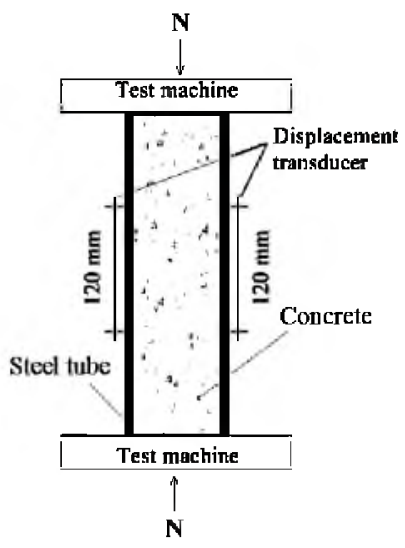

Fig. 1. Typical loading mode. 


\section{Results and Discussion.}

2.1. Mechanical Properties. It is easily derived from Figs. 2 and 3 that steel fibers can improve bending ductility of RPC significantly. The specimen containing $0.5 \%$ volume fraction of steel fibers showed a fragile fracture in testing. In contrast, the curve corresponding to the specimen containing $2 \%$ steel fibers shows a long and mild post-peak descending phase. However, steel fibers play a limited role in improving the ductility when $\mathrm{RPC}$ are subjected to compression. When compressive deformation passed its peak value corresponding to the peak stress, the loading-deformation curves of all specimens exhibited a drop followed by final fracture of the specimens. Unlike normal strength concrete (NSC), no relatively complete post-peak descending period can be captured for RPC, even at very low loading speeds. So it is not available to utilize steel fibers to increase ductility for compressive members of RPC.

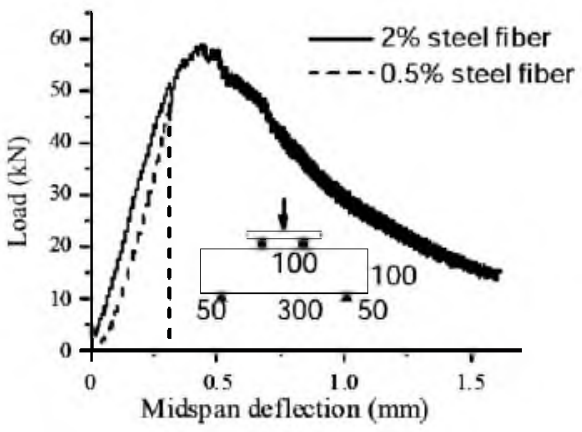

Fig. 2

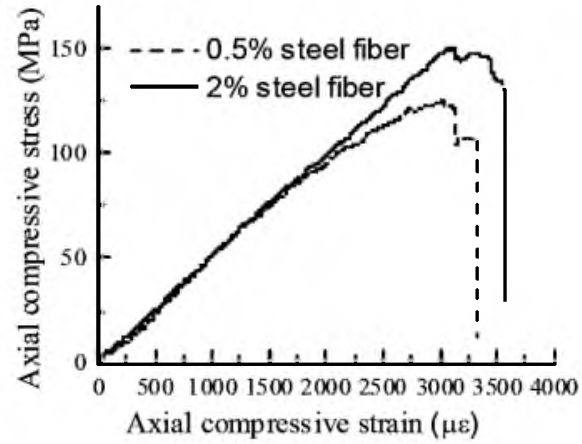

Fig. 3

Fig. 2. Four-point bending curves of RPC specimens.

Fig. 3. Stress-strain curves for RPCs under axial compression.

Poisson's ratio of RPC keeps constant (about 0.2) till compressive failure occurs (Fig. 4). Growth of Poisson's ratio is caused by the propagation of microcracks, which are mainly distributed in the interfacial transition zone (ITZ), a weak link that dominates strengths of concretes $[4,5]$. Low constant Poisson's ratio of RPC means that the ITZ is eliminated and, thus, the strength and ductility are improved.

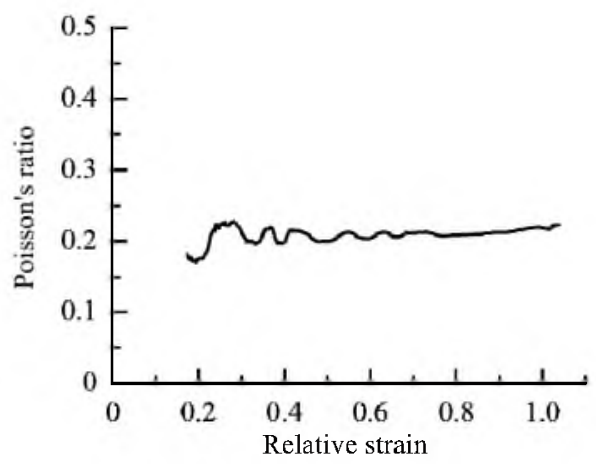

Fig. 4. Poisson's ratio vs. relative compressive strain of RPC.

The Young modulus of RPC measured in this study is $44 \mathrm{GPa}$. With higher strength but relatively lower Young modulus, RPC has much greater deformability than conventional concrete. The peak strain of RPC is $3000-3700 \mu \varepsilon$, which exceeds the yield strain of a common steel. Steel tube enters plastic stage when its compressive strain exceeds $1500 \mu \varepsilon$. 
It can be derived from a simple computation: when the longitudinal strain of an RPCFT column reaches $3000 \mu \varepsilon$ (approximately the peak strain of RPC), the lateral strain of the steel tube is about $1200 \mu \varepsilon$, yet the lateral strain of RPC is only about $600 \mu \varepsilon$. In another word, the steel tube enters plastic status too early and before the peak strain of RPC is reached, the incongruity in lateral deformation has cumulated to a significant extent.

As a conclusion, because properties of RPC are dramatically different from that of NSC, behavior of RPCFT columns differs from the normal CFT columns. The difference is reflected in two aspects: the load-strain curve shape and the calculation of bearing capacity. The following two sections focus in these two aspects separately.

2.2. Load-Strain Behavior of RPCFT Stub Columns under Uniaxial Compression. The typical load-strain curve of a RPCFT stub column (with or without steel fibers) under axial compression (Fig. 5) can be subdivided into the following four phases:

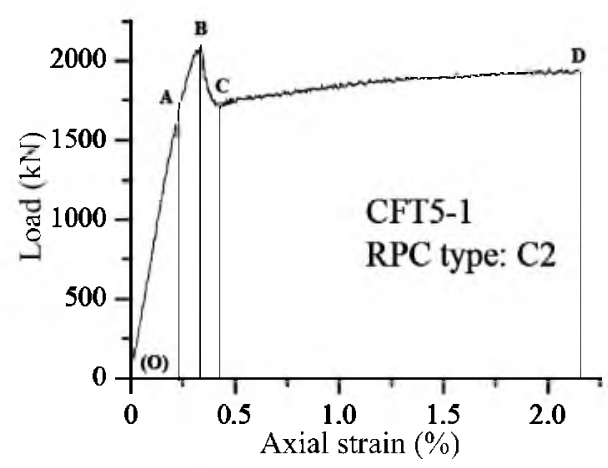

Fig. 5. A typical load-strain curve of fibered RPC filled steel tubular stub columns under axial compression.

(1) Linear Elastic Phase $(O A)$ : At this phase, both the steel tube and the core RPC correspond to the elastic stage, where the steel tube and core RPC operate independently.

(2) Elastic-Plastic Phase (AB): At this phase, the steel tube enters a plastic stage, the longitudinal rigidity of steel declines, leading to the reduction of the rigidity of the column. Meanwhile, Poisson's ratio of the steel tube increase quickly. However, core RPC is still at the elastic stage and its Poisson's ratio keeps constant because the critical deformability of RPC is greater than that of steel. As the lateral deformation of the steel tube is more than that of RPC, the steel tube and the core RPC still work independently, which is different from an NSC filled steel tubular column. When the load increases to point $B$, the ultimate bearing capacities of both core RPC and the RPCFT stub column are achieved.

(3) Descending Phase ( $B C)$ : When the ultimate load level is reached, the loading deformation curve descends rapidly. The failure mode of the core RPC casted in steel tubes is shear failure. To test this, when a specimen passed the point $B$ and the load is stopped immediately and extracted the PRC core (Fig. 6) via plasma cutting. There was a shear crack throughout the core, separating it into two wedges that can slip along the interface. If the loading continued, the two wedges would squeeze the tube wall and cause the confinement force from the tube wall, which tends to arrest the slip.

(4) Platform phase (CD): At this phase, the load-strain curve is horizontal. The confinement force from the tube wall is high enough to stabilize the slip. RPCFT stub columns exhibit excellent ductility. An RPCFT stub column still possesses relatively large bearing strength even when the longitudinal compression strain is up to $5 \%$.

The load-deformation behavior presents some similarities to the one noticed in concrete cylinders confined by unbonded, non-resin impregnated elastic fiber ropes (vinylon or polypropylene) of high tensile strain [6,7]. There temporary load drop was observed and finally load regaining. Despite the fact that the study concerns plain concrete 


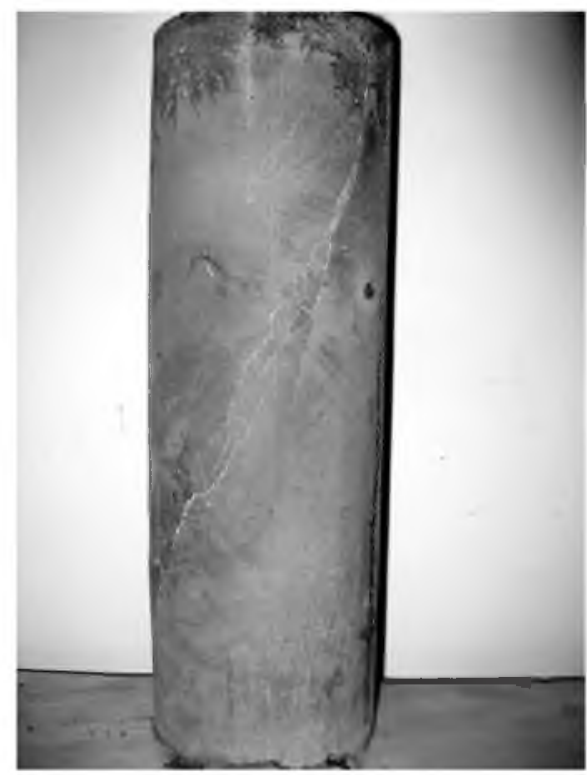

Fig. 6. Compressive failure mode of RPC in steel tubes.

externally confined with fiber ropes, it may share some common characteristics of the behavior of cracked RPC that interacts with an external steel jacket. Also, this behavior is characteristic for FRP-confined noncircular square columns, the temporary load drop and then load regaining has been observed in some cases.

2.3. Engineering Formula for Calculating the Axial Bearing Capacity of RPCFT Columns. Most formulas on the bearing capacity of CFT columns from literature and standards resemble the following equation:

$$
N_{c}=f_{c} A_{c}+\alpha f_{y} A_{s}
$$

where $N_{c}$ is the bearing capacity, $f_{c}$ is uniaxial compressive strength of RPC, $A_{c}$ is the cross section area of RPC, $f_{y}$ is the tensile yield strength of steel tube, $A_{s}$ is the cross section area of steel tube, and $\alpha$ is a factor not less than 1 to reflect the interactive extent, and it maybe 1.975 in European EC4 (1994), 1.7 in Chinese CECS 104:99 (1999), 1.27 in Japanese AIJ (1997) and 1 in American ACI (1999).

It is too early to draw any conclusions regarding which code is more accurate. Because the partial factors, the material testing methods, etc., vary in different countries, and some of the above codes take into account the long-term effects, such as concrete creep.

This study compares the $\alpha$ values reflected in these codes, in order to check their applicability to high-strength concrete. For long columns, the slenderness factor should be introduced into the formula for stub columns, however, this is beyond the scope of this paper.

The value of $\alpha$ derived by the least mean square regression of the test data is 1.4 . The comparison of the experimental data obtained via Eq. (1) for different $\alpha$ values is depicted in Fig. 7, where points located above the diagonal line imply that the respective calculation results overestimate the bearing capacity and vice versa. It is obvious that larger $a$ (such as in CECS and EC4) would lead to unsafe calculations.

The formula $N_{c}=f_{c} A_{c}+f_{y} A_{s}$, which is similar to the ACI code, is temporarily adopted for calculation of RPCFT columns, since it can guarantee their safe estimation. The interaction between steel tubes and concrete degrades with the strength of concrete. 

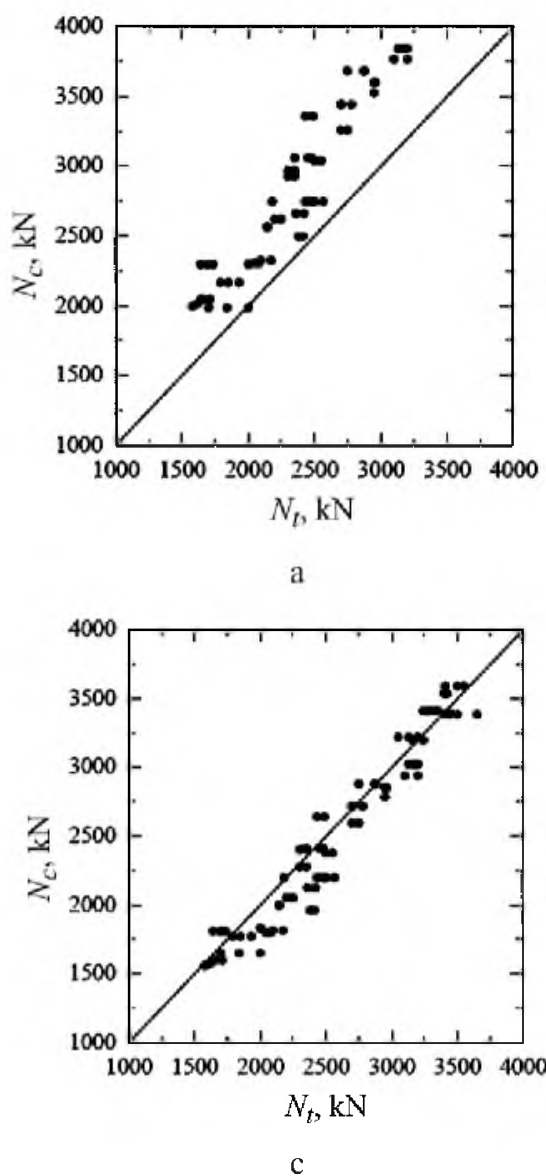

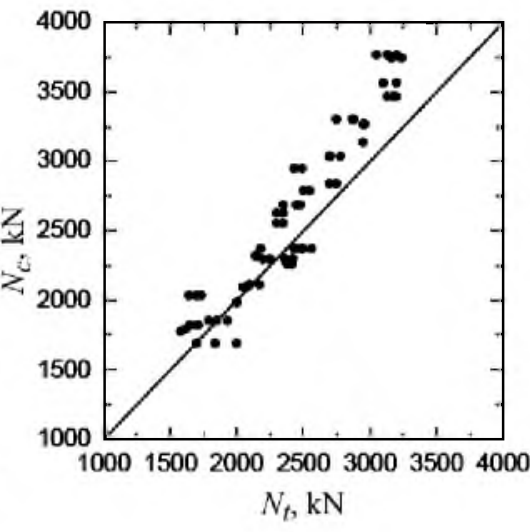

b

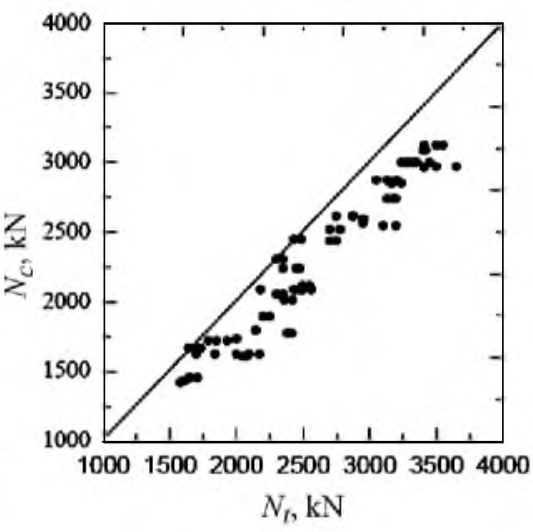

d

Fig. 7. Calculation results for different $\alpha$ values.

However, this disadvantage is trivial with respect to the fact that this type of columns enables the utilization of RPC in compression members.

Conclusions. RPCFT stub columns exhibit very high ductility and alleviate the RPC disadvantage of brittle failure. Steel tube confinement can improve the ductility of RPC, reducing the risk of collapse of structures resulted from fragile failure of RPC under high-stress conditions. Failure mode of RPCFT stub columns under axial compression is of shear type.

The formula $N_{c}=f_{c} A_{c}+f_{y} A_{s}$ is temporarily adopted for calculation of RPCFT columns, since it can guarantee their safe estimation. The interaction between steel tubes and concrete degrades with the strength of concrete.

Acknowledgments. This project is funded by the National Natural Science Foundation of China (51208391).

1. P. Richard and M. Cheyrezy, "Composition of reactive powder concretes," Cement Concrete Res., 25, No. 7, 1501-1511 (1995).

2. V. H. Perry and D. Zakariasen, "Overview of UHPC technology, materials, properties, markets and manufacturing," in: Proc. of the 3rd Int. Symp. on High Performance Concrete/PCI National Bridge Conference (October 19-22, 2003, Orlando, FL), Paper $60(2003)$. 
3. M. Alkaysi, S. El-Tawil, Z. C. Liu, and W. Hansen, "Effects of silica powder and cement type on durability of ultra high performance concrete (UHPC)," Cement Concrete Comp., No. 66, 47-56 (2016).

4. Y. Su, J. Li, C. Q. Wu, et al., "Effects of steel fibres on dynamic strength of UHPC," Constr. Build. Mater., 114, 708-718 (2016).

5. M. P. Collins, D. Mitchell, and J. G. MacGregor, "Structural design considerations for high strength concrete," Concr. Int. Des. Constr., 15, 27-34 (1993).

6. S. R. Razvi and M. Saatcioglu, "Strength and deformability of confined highstrength-concrete columns," ACI Struct. J., 91, No. 6, 678-687 (1994).

7. P. Y. Blais and M. Couture, "Precast, prestressed pedestrian bridge - world's first reactive powder concrete structure," PCI J., 44, No. 5, 60-71 (1999).

8. Z. Y. Lin, Research on Behavior of RPC Filled Circular Steel Stub Axial Columns, Dissertation, Fu Zhou University, Fuzhou, China (2004).

9. X. C. Pu, H. J. Pu, and Y. W. Wang, "Preparation and study on kilometer compressed material," Concrete, No. 3, 3-9 (2003).

10. P. Y. Yan and J. W. Feng, "Mechanical behavior of UHPC and UHPC filled steel tubular stub columns," in: E. Fehling, M. Schmidt, and S. Stürwald (Eds.), Ultra High Performance Concrete (UHPC), Proc. of the 2nd Int. Symp. on Ultra High Performance Concrete (March 5-7, 2008, Kassel, Germany), Kassel University Press $\mathrm{GmbH}$, Kassel (2008), pp. 355-362.

11. J. Zhang, Experiment Investigation on Behavior of Reactive Powder Concrete Filled Steel Stub-Columns, Dissertation, Fu Zhou University, Fuzhou, China (2003). 